\title{
Prácticas editoriales en materia de visibilidad de revistas científicas latinoamericanas en Ciencias Sociales y Humanas
}

Paola Sierra Flórez

Universidad Pontificia Bolivariana | paola.sierra@upb.edu.co / https://orcid.org/oooo-ooo2-4854-1143,

\author{
Maricela Gómez Vargas \\ Universidad Pontificia Bolivariana | maricela.gomez@upb.edu.co / https://orcid.org/oooo-0o03-4094-3145
}

\section{Resumen}

La investigación aborda las prácticas en materia de visibilidad que llevan a cabo las revistas científicas latinoamericanas y los factores influyentes. La técnica de recolección de información aplicada fue la encuesta. La muestra estuvo conformada por las revistas clasificadas en Scimago Journal o Country Rank (SJR), asociadas a cinco categorías temáticas: Filosofía, Teología, Derecho, Ciencias Políticas y Psicología. El principal resultado da cuenta del desaprovechamiento de las herramientas de la Web 2.0 por parte de las revistas analizadas, esto permite inferir que el posicionamiento de una revista no está directamente relacionado con el uso de este tipo de herramientas. A partir de los resultados obtenidos, se sugiere como línea futura de investigación la identificación de otros factores que incidan positivamente en la visibilidad de las revistas.

\section{Editorial Practices In Terms of Visibility Carried Out by Latin American Scientific Journals in Social Sciences and Humanities}

\footnotetext{
Abstract

This research addresses the editorial practices in terms of visibility carried out by Latin American scientific journals and their influential factors. The applied technique of data collection was surveyed. The sample was composed by journals classified in Scimago Journal er Country Rank (SJR), all of them associated to five subject categories

Palabras clave

Revistas científicas América Latina Visibilidad Impacto científico Ciencias Sociales Humanidades
Keywords

Scientific journals Latin American Visibility

Scientific impact Social Sciences Humanities 
in the Latin American context: Philosophy, Theology, Law, Political Science and Psychology. The main finding was that Web 2.0 tools are underused by the analyzed journals, we could infer that the positioning of a journal is not directly related with the usage of this kind of technological tools. From the results obtained, it is suggested, as a future research line, the identification of other factors that positively affect the visibility of the journals.

Artículo recibido: 1-11-2018. Aceptado: 21-05-2019

\section{Introducción}

La forma de comunicar la ciencia ha cambiado en los últimos años; hoy es más rápida, fluida, dinámica y participativa gracias al uso de las nuevas tecnologías. Es necesario que autores y editores tomen un papel determinante en los procesos de difusión de la ciencia que circula en las revistas con el fin de aumentar la visibilidad y el impacto de los contenidos publicados (Ruiz-Corbella y Galán-González, 2017; Fresco-Santalla, 2013; Robinson-García, Delgado-López-Cózar y Torres-Salinas, 2011).

Según González-Vegas (2010), la visibilidad es la posibilidad de que los artículos publicados en una revista sean hallados y leídos en un contexto tanto nacional como internacional. Por su parte, Ramírez-Martínez, Martínez-Ruiz y Castellanos-Domínguez (2012: 116), sostienen que la visibilidad de una revista es entendida como "la posibilidad que tienen los usuarios potenciales, en cualquier parte del mundo, de encontrar la información contenida en ella, a través de repositorios propios, institucionales o externos". A su vez, Colciencias (2016: 8), define la visibilidad internacional de una revista cuando es "incluida en diversos tipos de índices o bases, con lo cual se incrementa su aceptación en la comunidad científica respectiva".

Desde finales del siglo XX y principios del XXI, las revistas deben hacer frente al desafío que plantea el aumento desmedido de publicaciones y a la responsabilidad adjudicada al considerarlas como el principal medio de comunicación de la ciencia (Delgado-López-Cózar y Ruiz-Pérez, 2009). En este sentido, las revistas deben contar con altos niveles de visibilidad que les permita destacarse dentro de las múltiples posibilidades existentes. Villamón et al., (2005, citado por Miguel, 2011: 188) apunta que "cuanta más visibilidad tiene una revista, más interés despierta, mayor cantidad de trabajos recibe para su publicación y la selección de los artículos eleva la calidad de su contenido".

Para aumentar la visibilidad se precisa de las posibilidades que ofrecen las tecnologías de la Web 2.o, porque aumentan el rango de difusión de una publicación a un entorno mundial. Meredith (2010, citado por García Álvarez de Toledo y Fernández-Sánchez, 2011: 9), expresa que cuando un autor logra publicar un artículo en una revista, no puede pensar que su trabajo ha terminado, y resalta que "el camino no ha hecho más que empezar porque ese artículo sólo lo leerá una mínima parte del total de personas que podrían estar interesadas, incluidas muchas del ámbito científico".

La aplicación de la Web 2.0 en el ámbito científico implica asumir nuevas formas de comunicar y hacer investigación. Torres-Salinas (2008a), apunta que esta nueva forma de hacer las cosas supone un cambio más de carácter tecnológico que social, puesto que el investigador incorpora nuevas herramientas tecnológicas como apoyo a la labor científica en tres momentos: antes, durante y después (REBIUN, 2011).

El uso de tecnologías Web 2.0 ha empezado a cobrar importancia en estos ámbitos. Es posible encontrar una cantidad considerable de literatura sobre este tema; la mayoría 
evidencia una tendencia favorable hacia la implementación gradual de estas herramientas por parte de los autores y las revistas. Baiget (2009: 75) apunta:

la existencia de herramientas de comunicación más rápidas como los blogs, sitios web, feeds RSS y podcasts [...] aceleran la comunicación de la ciencia, pero circunvalan a las revistas. Los académicos pueden comunicarse por los nuevos canales y las revistas quizá deban tener un papel diferente del pasado, aunque sigue siendo indispensable que los hallazgos científicos queden registrados de una manera formal.

Según Tejada-Gómez (2012), las revistas científicas están sufriendo cambios asociados, entre otros factores, a lo digital; la migración de contenidos a la Web 2.0, las indexaciones, el open access, los repositorios, la aplicación de modelos económicos sostenibles, las normas de publicación, la profesionalización de la labor del editor y las prácticas éticas.

Torres-Salinas (2008a) afirma que el uso de tecnologías de la Web social o Web 2.0 permite a los investigadores compartir e intercambiar recursos, trabajar de forma cooperativa y difundir los resultados de investigación. Esto representa una oportunidad para constituir redes colaborativas de trabajo que pueden llevar a la producción de nuevos artículos científicos, así como la oportunidad de visibilizar la producción científica en otros escenarios sumado a que, las tecnologías Web 2.0, son un canal de comunicación alternativo para fortalecer el acceso a la producción científica por parte de un público más amplio y diversificado (Torres-Salinas y Delgado-LópezCózar, 2009).

Como se referencia en la literatura, los países de América Latina constituyen la periferia de la producción científica mundial. Piezzi (2010, citado en Álvarez-Rodríguez et al., 2017) denomina esta situación como "la ciencia perdida del tercer mundo". A pesar de que la región es una de las más activas en la creación de contenidos en acceso abierto, -aspecto que impacta positivamente en la visibilidad de las revistas (González-Vegas, 2010; Abadal, 2012)-y de que en la región se produce un gran número de revistas, éstas no han logrado la visibilidad y el impacto suficiente en el escenario internacional (Mendoza y Paravic, 2006; Miguel, 2011).

El objetivo principal de este trabajo es reconocer las prácticas en materia de visibilidad que incorporan algunas revistas científicas latinoamericanas de ciencias sociales y humanas, e identificar los factores que influyen o inhiben el posicionamiento de estas publicaciones.

\section{Metodología}

El diseño de esta investigación es de carácter cuanti-cualitativo. El universo de análisis estuvo compuesto por las revistas científicas clasificadas en Scimago Journal er Country Rank (SJR) con un cuartil recolectado en 2016. La selección de la muestra tuvo en cuenta los siguientes criterios:

1. Revistas asociadas a cinco categorías temáticas de las áreas de ciencias sociales y humanidades: Filosofía (Philosophy), Teología (Religius Studies), Derecho (Law), Ciencias Políticas (Political Science and International Relations) y Psicología (Psychology, miscellaneous).

2. Revistas procedentes de la región latinoamericana 
En total, la muestra quedó conformada por 83 revistas, sin embargo dos de ellas estaban incluidas simultáneamente en dos categorías temáticas (Filosofía y Ciencias Políticas y Filosofía y Teología, respectivamente), se decidió ubicar estas revista en las categorías con menor representación en la muestra, en este caso Ciencias Políticas y Teología, por lo que la muestra final quedó conformada por 81 revistas. La distribución por país, área y cuartil se describe en la tabla 1.

\begin{tabular}{|l|c|}
\hline País de procedencia & $\mathbf{\%}$ \\
\hline Brasil & 30,8 \\
\hline Colombia & 25,9 \\
\hline Chile & 17,2 \\
\hline México & 16 \\
\hline Venezuela & 3,7 \\
\hline Argentina & 2,4 \\
\hline Perú & 2,4 \\
\hline Puerto Rico & 1,2 \\
\hline & 99,6 \\
\hline
\end{tabular}

\begin{tabular}{|l|c|l|c|}
\hline Área del conocimiento & $\%$ & Quartil & $\%$ \\
\hline Psicología & 29,6 & $\mathrm{Q}_{4}$ & 53 \\
\hline Derecho & 27,1 & $\mathrm{Q}_{3}$ & 29,6 \\
\hline Filosofía & 23,4 & $\mathrm{Q}_{2}$ & 7,4 \\
\hline Ciencias Políticas & 16 & $\mathrm{Q}_{1}$ & 0 \\
\hline Teología & 3,7 & Sin quartil & 9,8 \\
\hline & & & 99,8 \\
\hline
\end{tabular}

Elaboración propia a partir de los datos proporcionados por Scimago Journal \& Country Rank en julio de 2016

Tabla 1. Caracterización de las revistas de la muestra

El instrumento de recogida de los datos fue la encuesta, la cual se aplicó por medio del formulario en línea de Google forms compuesto por 42 preguntas abiertas y cerradas. Se dirigió al director, editor, gestor, asistente o coordinador editorial o a alguno de los miembros del comité editorial de las revistas que conforman la muestra. La encuesta abordaba los siguientes aspectos: Edición, Difusión y visibilidad, Medición y factores influyentes. El primero pretendía identificar los procesos de convocatoria, recepción, diagramación y publicación llevados a cabo por las revistas. El segundo indagó por las estrategias de difusión y distribución, indexación en índices y bases de datos y la presencia en redes sociales. En la medición, se identificaron los indicadores utilizados por las revistas para medir el impacto, la influencia social y el uso. Por último, se detectan los factores que posibilitan u obstaculizan la visibilidad de las revistas científicas, tales como: apoyo institucional, soporte de publicación, condiciones óptimas, idioma y acceso abierto.

Para validar el instrumento, se realizó una prueba piloto con editores o expertos en el tema de la edición, la cual permitió realizar algunos ajustes y precisar la pertinencia de las preguntas.

La encuesta estuvo abierta por un periodo de dos meses, entre agosto y septiembre de 2017. Respondieron 35 revistas que representan el $43,2 \%$ del total de la muestra ( 81 revistas).

\section{Resultados}

En este apartado se identifican las prácticas en materia de visibilidad implementadas por las revistas y los factores que influyen en el posicionamiento de las publicaciones científicas. 


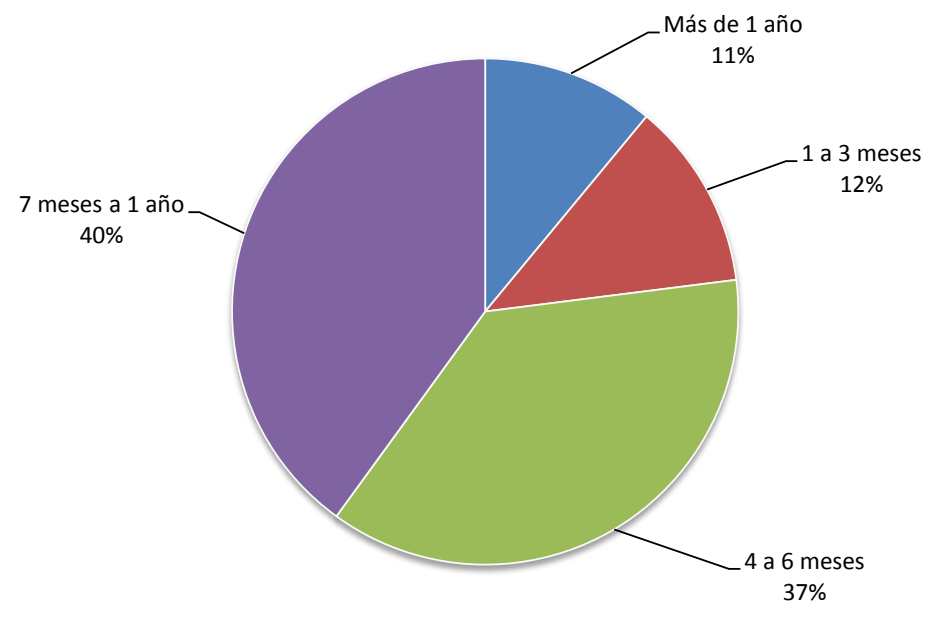

Fuente: elaboración propia

\section{Prácticas en materia de visibilidad de las revistas científicas}

Se presentan los resultados en relación a las prácticas implementadas para incrementar la visibilidad, a partir de tres aspectos: a) Edición, b) Difusión y visibilidad, y c) Medición.

\section{a) Edición}

Se describen los procesos de convocatoria, recepción, diagramación y publicación llevados a cabo por las revistas. En cuanto al primer aspecto, el $82,8 \%$ hace uso de herramientas automatizadas para realizar la convocatoria y recepción de los artículos. Específicamente, el 62,8\% utiliza el Open Journal Systems (OJS). Además, manifiestan la utilización de otras herramientas: Biblioteca Virtual en Salud (BVS), Saber UCV (Repositorio institucional de la Universidad Central de Venezuela), y los sistemas comerciales o de pago para la automatización del proceso editorial, entre los que mencionan: Editorial Express, EM - Editorial Manager, ScholarOne y Elsevier Editorial System (EES).

El 68,5\% indica que utiliza el sistema de gestión editorial para enviar los manuscritos, el $48,5 \%$ hace uso del correo electrónico, y varias revistas utilizan los dos medios de manera simultánea.

El $57,2 \%$ no establece ningún tipo de alianza o convenio, mientras que el $42,8 \%$ sí establece este tipo de relaciones. Los objetivos más recurrentes para establecer estas alianzas son la difusión de los contenidos, la participación de pares y, finalmente, la socialización de la convocatoria.

Aparecen dos rangos de tiempo como promedio de publicación de un nuevo número: entre cuatro y seis meses o entre siete meses a un año. En el gráfico 1 se presentan los resultados obtenidos en este aspecto.

Sobre los formatos para la publicación en línea de los contenidos, el preponderante es el PDF, usado por el 100\% de las revistas; el HTML, es el segundo más utilizado con el $58 \%$ y, el tercero, el XML con el $42 \%$.
Gráfico 1. Tiempo promedio de publicación 


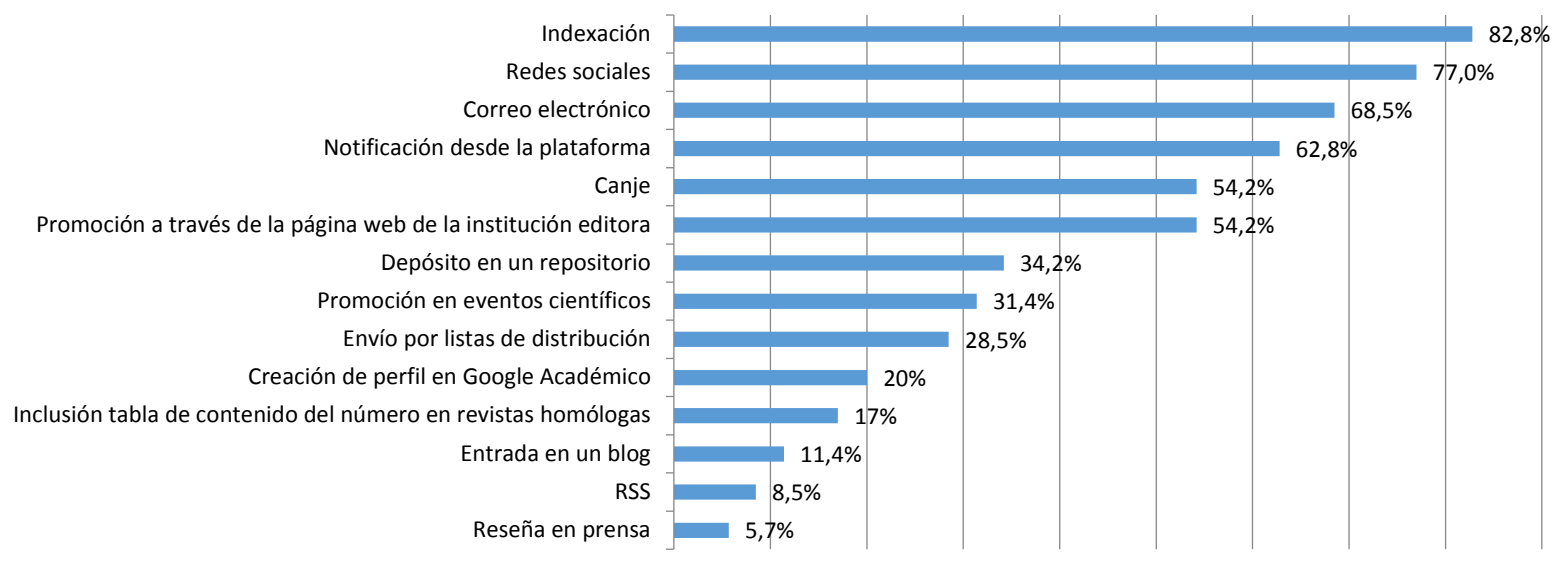

Gráfico 2. Estrategias de difusión y distribución

Fuente: elaboración propia

1. Acrónimo utilizado por el Departamento Administrativo de Ciencia, Tecnología e Innovación (Colciencias), en Colombia, el cual es utilizado para los procesos de evaluación, indexación y homologación de revistas especializadas.
Para la publicación de sus artículos lo habitual es el empleo simultáneo de dos o tres formatos. Las combinaciones más recurrentes son PDF/HTML/XML y PDF/HTML, cada una con el $28,5 \%$ respectivamente, el uso de PDF/XML representa el 14,2\% y hay un $25,7 \%$ que utiliza únicamente el formato PDF. En menor medida, utilizan otros formatos, en este caso, el EPUB, ISSUU y SGML. Por otra parte, el $66 \%$ no publica artículos en prensa (articles in press), esta estrategia permite circular los artículos de manera anticipada. Finalmente, el 82,8\% no genera contenido complementario.

\section{b) Difusión y visibilidad}

Aborda las estrategias de difusión y distribución, la indexación en índices y bases de datos, las orientaciones a los autores para la difusión y la presencia en redes sociales.

La indexación en bases de datos o en servicios o sistemas de indexación y resumen $\left(\mathrm{SIR}^{1}\right)$ es la principal estrategia para la difusión de los contenidos, con el 82,8\%. Las estrategias menos implementadas son la reseña en prensa con el 5,7\%, el RSS (contenidos sindicados) con el 8,5\% y la entrada en un blog, con el 11,4\%. El gráfico 2 muestra los resultados obtenidos en este aspecto. En esta pregunta los encuestados podían elegir más de una opción.

Otro aspecto muestra que el $88,5 \%$ permite a los autores difundir sus artículos a través de redes sociales y repositorios pero no orientan sobre cómo hacerlo. El 11,5\% restante, orienta en la forma de citar o referenciar el artículo, en la creación del perfil en el servicio gratuito Kudos, en la difusión en las redes sociales o perfiles académicos como ResearchGate o Google Académico.

En cuanto a las orientaciones brindadas a los autores sobre la normalización de su firma personal y afiliación institucional, el $54 \%$ no lo hace. Las revistas restantes proporcionan las siguientes indicaciones: 


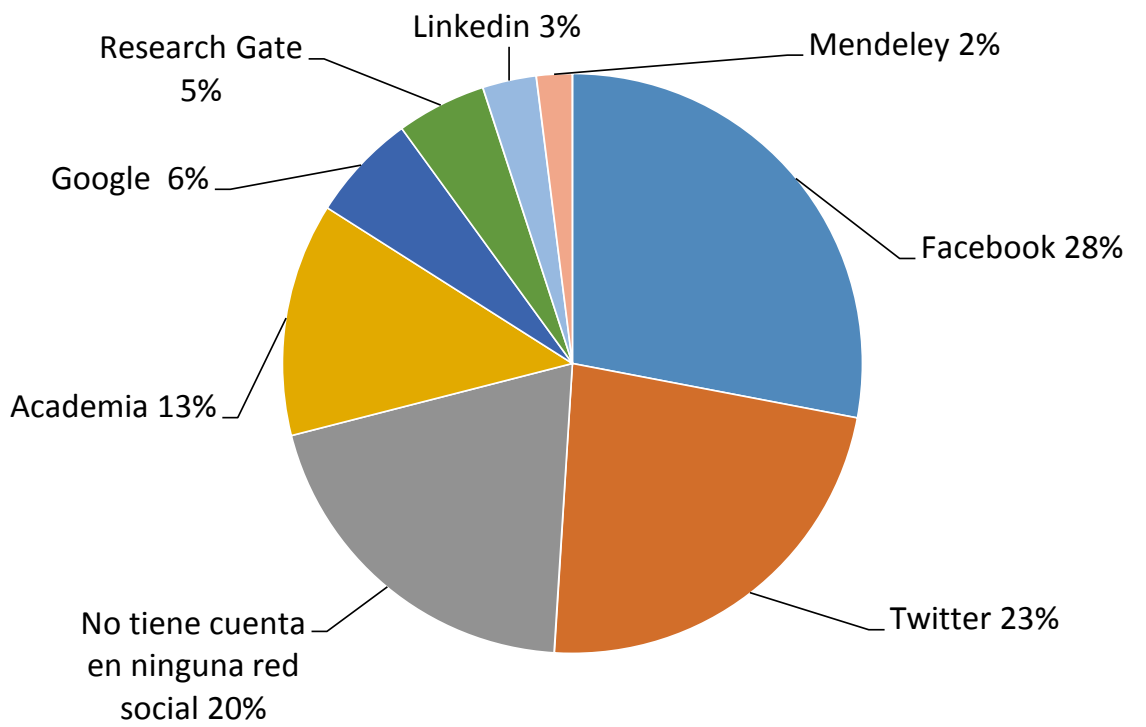

Gráfico 3. Medios sociales utilizados por las revistas

Fuente: elaboración propia

» Normalizar la afiliación institucional y el correo electrónico asociado a la institución.

"Contar con ORCID y perfil en Google Scholar.

" Incluir los datos y adscripción del autor en la primera nota al pie.

El identificador de objeto digital DOI (Digital Object Identification) es usado por el $74,2 \%$. Por otra parte, el 82,8\% cuenta con un plan de acción para la indexación en índices y bases de datos. Respecto a la presencia en redes sociales (Gráfico 3), es notorio que la herramienta de mayor uso es Facebook, seguida por Twitter.

\section{c) Medición}

Tiene en cuenta los indicadores utilizados para medir el impacto científico, la influencia social y el uso. En cuanto al primer grupo de indicadores, el $71,4 \%$ hace seguimiento a las citas que obtienen los artículos. Las herramientas más utilizadas para realizar este seguimiento son Scopus, con el 68,5\% y Google Académico, con el 51,4\%. En el gráfico 4 se presentan los resultados detallados. En este aspecto eran posibles varias opciones de respuesta.

Respecto a los indicadores de influencia social, el $80 \%$ indica que no hace seguimiento a este tipo de mediciones. $\mathrm{El} 20 \%$ restante que sí mide este tipo de indicadores y para ello hacen uso de los siguientes sistemas: Clarivate, Sistema interno de la institución editora, Google Analytics, Facebook, Altmetrics, Hootsuite y PlumbX.

El 82,2\% tienen acceso a estadísticas de visualizaciones y descargas de los artículos, y corresponde con aquellas que hacen uso del OJS. 


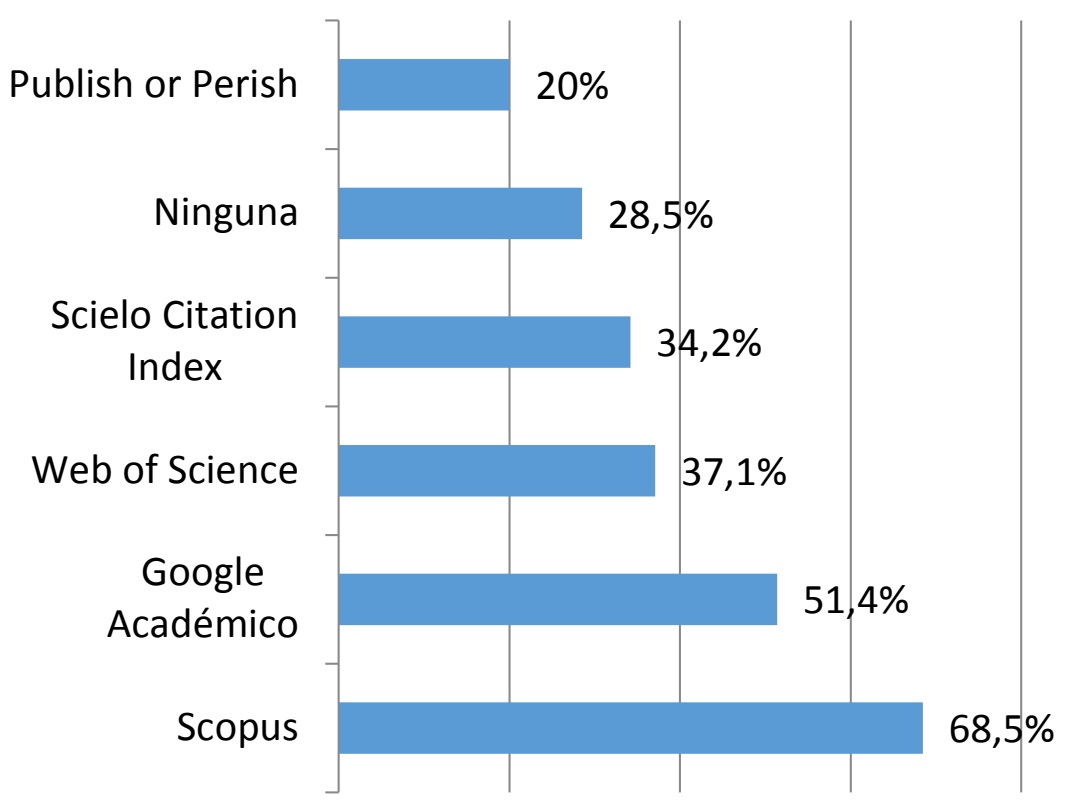

Fuente: elaboración propia

\section{Factores influyentes en el posicionamiento de las revistas científicas}

En este apartado se describen los resultados asociados a los factores que influyen en la visibilidad de las revistas científicas. Se tienen en cuenta los siguientes aspectos: a) Apoyo institucional, b) Soporte de publicación, c) Condiciones óptimas, d) Idioma y e) Acceso abierto.

\section{a) Apoyo institucional}

Aborda el aporte económico que brinda la institución editora y la asesoría, profesionalización y la disponibilidad de recursos (físicos, tecnológicos, humanos, de información y conocimiento, etc.) requeridos para la adecuada gestión de una revista. El $91,4 \%$ cuenta con este tipo de apoyo por parte de la institución editora.

\section{b) Soporte de publicación}

Se indaga sobre los soportes en los que se publican las revistas: impreso, digital o ambos. El 56\% opta por publicar simultáneamente en formato impreso y digital, mientras que el $44 \%$ lo hace solo en versión digital. En cuanto al impreso, ninguna publica exclusivamente de esta manera. El 87,5\% de las revistas que publica en digital cuenta con el apoyo técnico para la gestión de la herramienta.

\section{c) Condiciones óptimas}

Se consulta abiertamente sobre las condiciones óptimas que deben tener las revistas para lograr visibilidad en la Web. Los resultados son agrupados de la siguiente manera (Tabla 2): 


\begin{tabular}{|c|c|}
\hline Aspecto & Prácticas \\
\hline Formatos de publicación & $\begin{array}{l}\text { Usar varios formatos. } \\
\text { Publicar en formatos abiertos como XML. }\end{array}$ \\
\hline $\begin{array}{l}\text { Gestor electrónico de } \\
\text { archivos }\end{array}$ & Mejorar e incrementar el uso del OJS. \\
\hline Contenidos de calidad & $\begin{array}{l}\text { Cumplir con la periodicidad declarada. } \\
\text { Contar con autores conocidos. }\end{array}$ \\
\hline Infraestructura adecuada & $\begin{array}{l}\text { Mayor profesionalización y capacitación. } \\
\text { Personal suficiente. } \\
\text { Consejo editorial. } \\
\text { Buena gestión editorial. } \\
\text { Apoyo financiero y técnico. }\end{array}$ \\
\hline Indexación & $\begin{array}{l}\text { Indexación en bases de datos reconocidas y de alta } \\
\text { calidad, como Scopus, Web of Science, Scielo, etc. }\end{array}$ \\
\hline Acceso abierto & $\begin{array}{l}\text { Maximizar el uso de iniciativas abiertas de difusión del } \\
\text { conocimiento. } \\
\text { Incluir los contenidos en repositorios de acceso abierto. }\end{array}$ \\
\hline $\begin{array}{l}\text { Presencia en redes sociales } \\
\text { y en la Web }\end{array}$ & $\begin{array}{l}\text { Maximizar el uso de las redes sociales. } \\
\text { Realizar difusión por la Web. }\end{array}$ \\
\hline Sitio Web & $\begin{array}{l}\text { Contar con un sitio Web propio, moderno y atractivo. } \\
\text { Buen uso de metadatos. }\end{array}$ \\
\hline Idioma & Disponibilidad de los contenidos en múltiples idiomas. \\
\hline Estrategia digital & SEO (Search Engine Optimization), Adwords, etc. \\
\hline
\end{tabular}

Tabla 2. Condiciones óptimas para lograr visibilidad en la Web

Fuente: elaboración propia

\section{d) Idioma}

Se consulta si es posible considerar el idioma como un factor que limita la visibilidad de las revistas. El 51,4\% considera el idioma como un factor facilitador, mientras que el 48,6\% opina lo contrario. Uno de los argumentos de los editores frente a este tema es que el inglés es el idioma preponderante de la ciencia, lo que permite ampliar el alcance y la visibilidad. Además, expresan que las revistas que están mejor posicionadas, publican en este idioma. Sin embargo, algunos encuestados afirman que publicar en inglés no garantiza la visibilidad ya que el público objetivo de estas revistas son lectores de la región latinoamericana.

\section{e) Acceso abierto}

El $88,5 \%$ afirma que el open access es un factor que facilita la visibilidad y el impacto, porque democratiza el conocimiento, garantizando que sea de carácter público y abierto.

\section{Discusión}

Los resultados obtenidos apuntan a la agrupación de las prácticas de acuerdo con la frecuencia de implementación por parte de los editores de las revistas. Luego, se presentan los factores que influyen positiva o negativamente en la visibilidad. 
La práctica más estandarizada es el uso de un sistema automatizado de gestión editorial. Según la literatura, este tipo de herramientas permiten controlar, agilizar y hacer más eficiente el ciclo que inicia, de forma general, con el envío de un artículo, hasta que es publicado o rechazado (Jiménez-Hidalgo, Giménez-Toledo y Salvador-Bruna, 2008).

El sistema de gestión más empleado por las revistas de la región es el OJS, que coincide con los valores suministrados por Public Knowledge Project (2016) -organismo desarrollador de este sistema- y que indica que es el programa de gestión editorial más utilizado mundialmente, con 10.000 revistas activas, de las cuales, el 60\% pertenece a Latinoamérica. El OJS es un sistema robusto, que cuenta con las funcionalidades necesarias para realizar una adecuada gestión por parte de las revistas puesto que permite integrar y organizar todas las tareas a través del mismo espacio virtual y herramienta (García-Aretio, 2014).

Durante mucho tiempo, el correo electrónico fue el principal mecanismo para el envío de los artículos; no obstante, y gracias al constante avance tecnológico, este tipo de medios se ha ido tornando obsoleto. Con sistemas de gestión editorial como el OJS, es posible prescindir del correo electrónico y canalizar todo el proceso de recepción de los artículos a través de esta herramienta automatizada (Alfonso-Manzanet y Silva-Ayçaguer, 2014). Esto coincide con las prácticas que están implementado las revistas.

Estos sistemas de gestión permiten tener acceso a las estadísticas de uso y descarga de artículos, lo cual sirve de insumo a los editores para tomar acciones de mejora y orientar los procesos de gestión (Torres-Salinas y Delgado-López-Cozar, 2009). Diferentes autores recomiendan publicar las estadísticas en las páginas Web de las revistas, puesto que permiten conocer el uso real de los contenidos, y a los autores les da "una idea de la audiencia de los trabajos que hayan publicado, y a futuros autores del eco en lectores que se puede esperar de la revista, además de aportar transparencia a la publicación" (Abadal y Rius-Alcaraz, 2008: 253).

Las prácticas más comunes asociadas a los formatos de archivos de publicación coinciden con lo recomendado en la literatura en la que se sugiere hacer uso de formatos abiertos tanto para la recepción como para la publicación de los artículos, evitando problemas posteriores de lectura (Rojas-V. y Rivera-M., 2011). En este sentido, es favorable que utilicen en mayor medida formatos como PDF, HTML y XML.

Las revistas hacen uso de la indexación, como principal estrategia de difusión y distribución. Tradicionalmente han incorporado esta práctica debido a la creencia generalizada que considera la inclusión en base de datos como un indicador de calidad de las mismas, ya que para que ello ocurra, éstas deben cumplir con altos estándares de calidad. En países como España, contar con una amplia indexación en bases de datos es un factor de calidad, sobre todo, si la indexación es en Science Citation Indexy Social Science Citation Index (Delgado-López-Cózar, Ruiz-Pérez y Jiménez-Contreras, 2006). Con las dinámicas actuales de la comunicación científica, las revistas deben considerar los canales tradicionales de difusión y distribución y empezar a incorporar medios alternativos que permitan una cobertura más amplia y diferenciada de los contenidos (Torres-Salinas y Delgado-LópezCózar, 2009; García-Álvarez de Toledo y Fernández-Sánchez, 2011; Fresco-Santalla, 2013).

Los resultados demuestran un uso incipiente de las redes sociales. Las revistas que han adoptado estas herramientas hacen uso principalmente de Facebook y Twitter. Este resultado encuentra una relación similar con los hallazgos obtenidos por Camargo-Camargo (2016) y Veiga de Cabo y Martin-Rodero (2011), donde estas redes también son las más utilizadas, pero con porcentajes muy bajos. Además, las revistas están haciendo uso de las redes sociales generalistas, dejando de lado herramientas enfocadas al campo científico o académico, como Mendeley, Academia y ResearchGate. 
En la literatura se encuentran casos de revistas científicas que apuntan tanto a las redes sociales de carácter general como a las enfocadas al campo científico. Es el caso de la revista Comunicar, que tiene presencia en Facebook, Twitter, LinkedIn y en ResearchGate, Mendeley y Academia; de forma adicional tiene presencia en Youtube y Flickr. La revista El Profesional de la Información también utiliza estas mismas redes de carácter general, pero adicionalmente utiliza Pinterest.

Sin embargo es conveniente resaltar la posición de García Álvarez de Toledo y FernándezSánchez (2011), quienes afirman que las redes sociales generalistas no son sitios para debatir cuestiones científicas profundas, aunque es posible que se presenten este tipo de situaciones; lo cierto es que no se puede negar la utilidad que tienen en términos de divulgación y promoción de contenidos.

Respecto a los indicadores de impacto, los resultados permiten evidenciar que las revistas hacen seguimiento a las citas que obtienen a través de las bases de datos citacionales de mayor tradición como Scopus y Web of Science.

Otra práctica utilizada comúnmente para aumentar la visibilidad es la definición de políticas editoriales para que los autores puedan emprender acciones de difusión de los artículos en las redes sociales o para depositarlos en repositorios institucionales. Hay una actitud de apertura en este sentido, porque la mayoría permite que los autores difundan ellos mismos sus obras a través de las redes sociales u otros sitios. Sin embargo, no les brindan suficientes indicaciones para difundir sus publicaciones; cuestión que las revistas deberían fortalecer si tienen en cuenta que esta práctica conlleva un impacto positivo tanto en la revista como en los autores (Aparicio, Banzato y Liberatore, 2016). La literatura señala el caso de la revista El Profesional de la Información, la cual envía a sus autores los PDF definitivos para su libre e inmediata difusión y les recomiendan que, a su vez, ellos lo envíen a los autores que citaron en el artículo con el objetivo de ayudar a aumentar el Factor de impacto (IF) y el SCImago Journal Rank (SJR) de la revista (Baiget, 2012).

En cuanto a las prácticas menos desarrolladas por las revistas, los editores no suelen establecer alianzas o convenios de trabajo con publicaciones homólogas o asociaciones científicas, aspecto que impacta de forma negativa en la gestión de la revista. Estas acciones benefician la gestión en aspectos asociados a la difusión de la convocatoria y los contenidos, además de que facilitan la consecución de pares evaluadores. De la misma forma, contribuyen al enriquecimiento de estrategias y experiencias de trabajo, lo que redunda en la profesionalización de la labor editorial (Álvarez-Rodríguez et al., 2017; Ramírez-Martínez et al., 2012).

Según los resultados, las revistas no están implementado la publicación de artículos en prensa (article in press). La mayoría realizan prácticas tradicionales porque esperan reunir la cantidad necesaria de artículos para conformar un nuevo número, dejando de lado la publicación de los artículos a medida que son aprobados. La literatura señala como buena práctica el caso de la revista española Comunicar, que implementa esta estrategia permitiendo un mayor dinamismo e impactando positivamente en los autores, al tiempo que contribuye a incrementar la visibilidad de la revista (Aguaded-Gómez y FonsecaMora, 2012).

Las revistas participantes de este estudio no tienen prácticas definidas para orientar a los autores en cuanto a la normalización de su firma personal y afiliación institucional. Los referentes permiten corroborar que es importante ofrecer guías al respecto, puesto que esto afecta la visibilidad y dificulta la recuperación de las publicaciones y de las citas recibidas (Alonso-Arévalo, 2016). Es por esta razón, que países como España, realizan iniciativas para garantizar que los autores firmen de forma adecuada. Por ejemplo, la Fundación Española para la Ciencia y la Tecnología (Fecyt) estableció una 
serie de recomendaciones para definir la firma y la revista El Profesional de la Información, el repositorio E-LIS, la Universidad de Barcelona y la Universidad Politécnica de Valencia, pusieron en marcha la Web IraLIS (International Registry of Authors -Links to Identify Scientists) para concienciar a los investigadores sobre "algo tan sencillo pero tan importante como es la firma" (Baiget y Torres-Salinas, 2013: 27).

En cuanto al uso de identificadores únicos como el ORCID, los resultados indican que no emplean esta herramienta, aspecto que afecta negativamente tanto a las revistas como a los autores, teniendo en cuenta que este recurso además de resolver el problema de identificación unívoca de la autoría, permite vincular las actividades de investigación y sus productos (Aparicio et al., 2016). Es una herramienta clave para el seguimiento de las métricas en los autores (Altmetrics), ya que recopila datos a través de plataformas como Altmetric.com, Plum Analytics e ImpactStory.org, o de redes sociales académicas como ResearchGate.net, Academia.edu, GetCited.org (AlonsoArévalo, Cordón-Garcia y Maltrás Barba, 2016).

En cuanto a la generación de contenido complementario, las revistas hacen poco uso de esta estrategia. Torres-Salinas (2008b), señala que este tipo de material está dirigido principalmente a un público más profesional, amplía el espectro de lectores de la revista e introduce una comunicación directa con el lector. En la literatura se señalan ejemplos de revistas que implementan esta práctica, entre ellas se encuentran Plos, Comunicar y El Profesional de la Información. En el caso concreto de la revista Comunicar, se genera material complementario en forma de videos, los cuales comparte a través de su canal en YouTube, en el cual publica entrevistas, mesas redondas y sugerencias para la presentación de manuscritos o donde se explica el proceso de selección de los mismos (Aguaded-Gómez y Fonseca-Mora, 2012).

La mayoría no suelen medir la influencia social a través de las métricas alternativas; posiblemente por la falta de conocimiento sobre el tema, lo que se puede inferir de acuerdo a algunas de las respuestas proporcionadas. Las pocas que hacen seguimiento de la influencia social utilizan instrumentos como Google Analytics (herramienta de analítica Web que ofrece de forma gratuita Google). Aunque su uso es generalizado en el ámbito empresarial y especialmente en el comercio electrónico. La literatura indica que las revistas la usan para monitorear el comportamiento de su sitio Web (Maricato y Fernandes, 2015; Uribe-Tirado, Vallejo-Echavarría y Betancur-Marín, 2016; Abadal y Rius-Alcaraz, 2008).

Los factores que influyen de forma positiva en la visibilidad de las revistas científicas son: el uso del soporte digital, el papel del consejo editorial, el posicionamiento Web, la inclusión de contenidos en un segundo idioma, el acceso abierto, la atracción de autores reconocidos y el apoyo de la institución editora.

Las revistas hacen uso de soportes impresos y digitales de manera simultánea. Esta es una práctica que poco a poco tiende a desaparecer debido a las múltiples ventajas que permite la edición digital. Una de ellas es la mayor cobertura que se logra si se compara con la que puede obtener una edición impresa, puesto que está limitada al tiraje y a los convenios de canje establecidos con otras instituciones (Aparicio et al., 2016). Adicionalmente, el soporte digital facilita el acceso simultáneo de un mismo contenido por parte de varios lectores, sin importar el tiempo y el lugar. Sumado a lo anterior, las alternativas de edición aumentan significativamente, permitiendo incorporar en los artículos material complementario como videos, fotografías, tablas, mapas, gráficos, entre otros. En este orden de ideas, la edición digital de una revista es considerada como un factor facilitador al tener en cuenta que permite adoptar prácticas que garantizan la visibilidad y difusión de los contenidos. 
Por su parte, el consejo editorial es clave para el funcionamiento de una revista científica ya que permite asegurar el control de calidad de los contenidos, y debe estar conformado por científicos distinguidos con amplia trayectoria. Su tamaño y composición es tomado además como un indicador indirecto del prestigio de una revista, puesto que ponen de manifiesto la capacidad de atraer a científicos reputados y de abrirla al exterior. Sin embargo no basta con contar con un notable consejo editorial para dar una imagen positiva a la revista, este debe ser funcional (Delgado-LópezCózar et al., 2006).

Con la incorporación de las revistas científicas en el entorno digital, estas adaptan técnicas provenientes de otros ámbitos y les permiten lograr mayor visibilidad. Por ejemplo, el SEO es una estrategia de posicionamiento en la Web, la cual optimiza los resultados que arrojan los motores de búsqueda, al aparecer en los primeros resultados (Codina, 2017). Las revistas están incorporando algunas prácticas de SEO, pero aún son incipientes.

Otro factor que incide positivamente en la visibilidad es la publicación en un segundo idioma. Hay una tendencia generalizada a priorizar el inglés como lengua preponderante, incluso para las ciencias sociales y humanas, aunque es un tema controversial en el contexto iberoamericano porque se tiende a asumir una actitud de defensa hacia las lenguas nativas; una temática que es objeto de discusión en cuanto a la pertinencia y el impacto local versus la pertinencia y el impacto global (Aparicio et al., 2016; Ramírez-Martínez et al., 2012).

Como lo refiere la literatura, el idioma de publicación de un artículo determina el área geográfica donde puede tener impacto y el inglés es el idioma más usado del mundo. Para que una revista pueda incrementar su visibilidad mundialmente debe considerar la publicación de contenidos en este idioma (Baiget y Torres-Salinas, 2013). Como complemento a lo anterior, Aréchaga (2012) señala que no incorporar un idioma universal como lo es el inglés, puede ocasionar inconvenientes relacionados con la gestión de las revistas, cuando se trata, por ejemplo, de buscar evaluadores competentes para la mayoría de las áreas de investigación.

La mayoría de las revistas participantes ven el acceso abierto como un aliado para incrementar la visibilidad de los contenidos que publican. Las razones que adjudican están relacionadas con aspectos que son comúnmente mencionados en la literatura. El acceso abierto elimina las barreras de tipo económico, geográfico y social, lo que permite la democratización del conocimiento. La calidad de la investigación se ve favorecida, tanto en términos cualitativos como cuantitativos, puesto que los investigadores pueden acceder de forma rápida y ágil a los contenidos que requieren para la realización de sus investigaciones. Además, el acceso abierto constituye la oportunidad perfecta para que las pequeñas revistas, que no han tenido cabida dentro del mercado editorial tradicional, encuentren un espacio, puesto que el dominio deja de estar marcado por las grandes editoriales (Abadal, 2012).

Otro factor facilitador es atraer autores reconocidos. Algunos autores afirman que los investigadores tienden a publicar en revistas que cuentan con prestigio y posicionamiento en los principales índices de citación (Delgado-López-Cózar et al., 2006). Para una revista científica de la región, es un reto atraer a los mejores autores. Ante la falta de interés por parte de los autores por publicar en revistas con poca visibilidad, las revistas optan por la práctica de solicitar artículos a investigadores reconocidos, que a su vez contribuyan a aumentar el reconocimiento de la revista (Buela-Casal, 2002, citado por Torres-Salinas, 2010). 
La mayoría de las revistas encuestadas provienen del ámbito universitario, condición que trae consigo características particulares, razón por la cual el apoyo por parte de la institución editora es aún más importante. Al respecto Aguirre (1998, citado en Mendoza y Paravic, 2006: 63) sostiene que:

Es una realidad que cuando las revistas científicas son el producto del trabajo editorial de un grupo de académicos pertenecientes a cualquier unidad universitaria, cada edición es casi siempre de forma artesanal, ocupando ratos libres y si existen unidades centrales de producción editorial universitaria, éstas son pequeñas, con escasos medios materiales y/o profesionales, situaciones que impiden salidas de volúmenes a una periodicidad adecuada y evitan que las revistas ofrezcan un espacio dinámico de discusión, réplica y contestación, modalidades necesarias en la vida científica.

Por lo anterior, las revistas del contexto latinoamericano requieren de un compromiso decidido por parte de la institución editora, al procurar siempre que las publicaciones cuenten con todos los recursos necesarios para garantizar su correcto funcionamiento.

Los factores que inhiben la visibilidad de las revistas son: la escasa profesionalización, el financiamiento y el recurso humano dedicado a la edición. Según lo expresado por Ramírez-Martínez et al., (2012), las revistas científicas entendidas como un proyecto serio, requieren de recursos de toda índole que permitan su correcto funcionamiento. De acuerdo con la posición de estos autores algunos de los recursos necesarios para gestionar adecuadamente una revista científica son: recursos físicos, recursos de información y conocimiento, recursos económicos y de gestión, recursos humanos y recursos de infraestructura.

La literatura hace mención igualmente a problemas relacionados con la profesionalización tanto del editor como de su equipo de trabajo y por lo tanto del proceso en general (Ramírez-Martínez et al., 2012; Delgado-López-Cózar et al., 2006). En algunos casos la labor del editor es asumida por docentes-investigadores, los cuales, si bien pueden dominar el área temática de la revista, carecen del conocimiento y la experticia necesaria para desempeñar la labor editorial (Aparicio et al, 2016). Para mitigar un poco esta dificultad, en algunos países, instituciones adscritas por lo general al gobierno, apoyan la formación de editores y la profesionalización, estandarización y visibilidad de las revistas científicas (Ramírez Martínez et al., 2012). Este caso se presenta por ejemplo de países como España, por medio de la Fundación Española para la Ciencia y la Tecnología (Fecyt) o en Argentina con el Centro Argentino de Información Científica y Tecnológica (CAICYT) (Aparicio et al., 2016; Fundación Española para la Ciencia y la Tecnología, 2012).

En general, la literatura refiere dificultades relacionadas con estos aspectos, especialmente en la región latinoamericana, donde, como se mencionó anteriormente, dicha gestión editorial es asumida principalmente por las universidades; situación que también se presenta en países como España (Corera-Álvarez y Molina-Molina, 2016).

A partir de los cambios que trae consigo la incorporación de las nuevas tecnologías en la gestión de una revista científica, es necesario que esta tarea se apoye en profesionales con la formación y las capacidades específicas para ello. Al respecto, RodríguezYunta y Tejada-Artigas (2013), señalan que se requiere una nueva mentalidad en el equipo editorial que implique "pensar en digital" y en ese sentido la sugerencia es que la profesionalización además de los conocimientos inherentes a la edición de revistas científicas esté encaminada a aspectos de carácter tecnológico propios del entorno digital, entre ellos: XML, metadatos, formatos enriquecidos, sistemas automatizados de gestión editorial como el OJS, nuevos formatos para la lectura en dispositivos 
móviles, uso de las redes sociales, plataformas editoriales, bases de datos, portales científicos, blogs, etc. Todo esto para mejorar el posicionamiento de las revistas en la Web y presencia en los buscadores.

\section{Conclusiones}

Existe un desaprovechamiento de las herramientas de la Web 2.0 por parte de las revistas objeto de esta investigación, tanto para promover la interacción y la participación, como para divulgar, crear comunidad y estimular la creación de redes. Lo anterior se puede entender a partir de la insuficiente asignación de recursos con la que cuentan las revistas participantes, ya que la mayoría provienen del contexto universitario donde los recursos son limitados.

Las revistas han asumido de manera generalizada el concepto del Open Access, debido al uso extendido del OJS como herramienta de gestión, pero en especial como medio para obtener visibilidad Web y facilitar el acceso a los contenidos. Esto indica una clara intención de apertura, pero aún falta por fortalecer otros aspectos como estimular la interacción entre los actores (pares, autores, lectores, editores) y la publicación de contenidos complementarios que sean accesibles y aporten al movimiento denominado Open Data.

A pesar de lo anterior, es notorio que los editores no han adoptado estrategias para orientar y acompañar a los autores en la fase de difusión de los contenidos. Es oportuno hacer ajustes en las políticas editoriales y brindar recomendaciones a los autores para que gestionen la difusión, visibilidad e impacto de sus artículos. Una forma de hacerlo es generando contenidos didácticos que guíen en la creación de perfiles en las redes sociales académicas e instructivos para difundir a través de las páginas Web personales o repositorios institucionales. Al mismo tiempo, es necesario capacitar al equipo editorial en temas asociados al uso y gestión de herramientas como los identificadores únicos tanto para los artículos como los autores, redes sociales académicas y métricas.

La práctica de publicación de contenidos en soporte impreso tiende a desaparecer, lo que ha llevado a publicar en versión digital. Este uso facilita los procesos editoriales y los medios para obtener mayor visibilidad.

Las revistas presentan una apertura en cuanto al idioma de publicación considerándolo como un factor facilitador, puesto que permite incrementar los niveles de internacionalización y visibilidad de las revistas.

Se percibe cierto desconocimiento en la gestión editorial por parte de los responsables de su edición, por lo que se requiere profesionalizar la labor editorial, en aspectos propios a la gestión de una revista científica, así como en asuntos relacionados con la incorporación de herramientas tecnológicas del entorno digital.

Los resultados permiten inferir que la Web 2.0 no es una herramienta imperativa para la visibilidad e impacto de las revistas científicas, a pesar de que las revistas analizadas cuentan con un posicionamiento positivo, no implementan estrategias e instrumentos que facilitan la difusión y gestión de los contenidos. A partir de lo anterior, se propone como línea futura de investigación la identificación de otros factores que inciden directamente en la visibilidad e impacto de las revistas, tales como: los niveles de desarrollo de las comunidades académicas que soportan las revistas, los avances científicos de las áreas, la disposición de los investigadores para comunicar, entre otras. 


\section{Agradecimientos}

Las autoras agradecen el apoyo de las personas que atendieron la invitación a participar en la encuesta ya que su intervención fue fundamental para el desarrollo de esta investigación. 


\section{Referencias bibliográficas}

》 Abadal, Ernest. 2012. Acceso abierto a la ciencia. Barcelona: Editorial UOC. (Colección El Profesional de la Información). <http://eprints.rclis.org/16863/1/2012acceso-abierto-epi-uoc-vfinal-autor.pdf> [Consulta: 11 agosto 2017].

"Abadal, Ernest y Lluís Rius-Alcaraz. 2008. Revistas científicas de las universidades españolas: acciones básicas para aumentar su difusión e impacto. En Revista Española de Documentación Científica. Vol. 31, no. 2, 242-262.<https:// doi.org/10.3989/redc.2008.v31.i2.427>.

" Aguaded-Gómez, Ignacio y M. Carmen Fonseca-Mora. 2012. Ejemplos de buenas prácticas en la edición de revistas científicas: Comunicar. En Fundación Española para la Ciencia y la Tecnología, ed. Manual de buenas prácticas en edición de revistas científicas. España: Fecyt. p. 11-19. <https://www.revistacomunicar. com/pdf/2013-guia-buenas-practicas.pdf> [Consulta: 5 julio 2017].

»Alfonso-Manzanet, Jose Enrique y Luis Carlos Silva-Ayçaguer. 2014. Gestión automatizada en el proceso editorial de una revista científica como demanda inaplazable para favorecer la cultura comunicacional. En Educación Médica Superior. Vol. 28, no. 1, 145-153. <http://scielo.sld.cu/scielo.php?script=sci_arttext\&pid=So86421412014000100015\&lng=es\&nrm=iso > [Consulta: 1 octubre 2017].

"Alonso-Arévalo, Julio. 2016. Cómo gestionar la visibilidad e impacto de la investigación. <https://es.slideshare.net/jalonsoarevalo/como-gestionar-la-visibilidad-de-la-investigacin > [Consulta: 1 octubre 2017].

»Alonso-Arévalo, Julio; José Antonio Cordón-García y Bruno Maltrás-Barba. 2016. Altmetrics: medición de la influencia de los medios en el impacto social de la investigación. En Documentación Multimedia. Vol. 27, no. 1, 75-101.<http:// dx.doi.org/10.5209/rev_CDMU.2016.v27.n1.52870>

»Álvarez-Rodríguez, Dayana; Norbisley Fernández-Ramírez; Ernesto Piñero de Laosa; Lireima Risco-Villamañán; Florentino Uña-Izquierdo y José Alberto Bertot. 2017. Estrategia de difusión para la Revista de Producción Animal. En Revista de Producción Animal. Vol. 29, no. 1, 1-6. <http://scielo.sld.cu/scielo. php?script=sci_arttext\&pid=S2224-79202017000100001\&lng=es\&tlng=es $>$ [Consulta: 1 octubre 2017].

" Aparicio, Alicia; Guillermo Banzato y Gustavo Liberatore. 2016. Manual de gestión editorial de revistas científicas de Ciencias Sociales y Humanas. Buenos Aires: CLACSO: CAICYT-CONICET: PISAC: Consejo de Decanos. Facultades de Ciencias Sociales y Humanas: Ministerio de Ciencia y Tecnología MINCyT: Ministerio de Educación y Deportes: REUN - Red de Editoriales de Universidades Nacionales. <http://biblioteca.clacso.edu.ar/clacso/se/20170209122904/ Manual_PISAC.pdf> [Consulta: 1 agosto 2017].

"Aréchaga, Juan. 2012. The International Journal of Developmental Biology. En Fundación Española para la Ciencia y Tecnología, ed. Manual de buenas prácticas en edición de revistas científicas. España: Fecyt. p. 21-25. <https://www.revistacomunicar.com/pdf/2013-guia-buenas-practicas.pdf> [Consulta: 6 septiembre 2017].

» Baiget, Tomás. 2009. Nuevas vías de comunicación científica experimentadas desde una revista. En Ibersid, 75-80. <http://eprints.rclis.org/20054/1/Nuevasvias-comunicacion-revista-cientifica.pdf>[Consulta: 27 julio 2017]. 
》 Baiget, Tomás. 2012. Ejemplos de buenas prácticas en la edición de revistas científicas: El Profesional de la información (EPI). En Fundación Española para la Ciencia y Tecnología, ed. Manual de buenas prácticas en edición de revistas científicas. España: Fecyt. p. 33-43. <https://www.revistacomunicar.com/pdf/2013-guia-buenas-practicas. pdfs [Consulta: 6 septiembre 2017].

" Baiget, Tomás y Daniel Torres-Salinas. 2013. Informe APEI sobre publicación en revistas científicas. Gijón: Asociación Profesional de Especialistas en Información. <https://www.um.es/documents/793464/1180186/InformeAPEI-Publicacionescientificas2ed.pdf/7bf25ofo-3bfo-4C47-9a8a-89ad5437c138> [Consulta: 6 septiembre 2017].

»Camargo-Camargo, María Claudia. 2016. Adopción y Uso de Herramientas Digitales por Revistas Académicas de Comunicación en Iberoamérica. Barranquilla: Universidad del Norte, 2016. 112 p. Tesis de maestría. <http://manglar.uninorte.edu.co/ bitstream/handle/10584/5813/1140826073.pdf?sequence $=1 \&$ isAllowed $=y>$ [Consult a: 15 mayo 2017].

"Codina, Lluis. (2017). SEO académico: definición, componentes y guía de herramientas. [Mensaje en un blog]. <https://www.lluiscodina.com/seo-academicoguia/\#guia>[Consulta: 1 octubre 2017].

"Colciencias. 2016. Política para mejorar la calidad de las publicaciones científicas nacionales. Colciencias. <http://colciencias.gov.co/sites/default/files/upload/paginas/ politica-publindex-colciencias.pdfs [Consulta: 1 febrero 2017].

» Corera-Álvarez, Elena y Silvia Molina-Molina. 2016. La edición universitaria de revistas científicas. En Revista Interamericana de Bibliotecología. Vol. 39, no.3, 277-288. $<$ https://doi.org/10.17533/udea.rib.v39n3ao5>

»Delgado-López-Cózar, Emilio y Rafael Ruiz-Pérez. 2009. La comunicación y edición científica: fundamentos conceptuales. En Homenaje a Isabel de Torres Ramírez: estudios de documentación dedicados a su memoria. Granada: Universidad de Granada. p. 131-150. <http://eprints.rclis.org/13988/1/ Emilio_Delgado_Lopez_Cozar_y_Rafael_Ruiz_La_comunicacion_y_edicion_cientifica_fundamentos_conceptuales_Granada_2009.pdf> [Consulta: 1 agosto 2017].

»Delgado-López-Cózar, Emilio; Rafael Ruiz-Pérez y Evaristo Jiménez-Contreras. 2006. La edición de revistas científicas. Directrices, criterios y modelos de evaluación. Granada: Universidad de Granada. <https://www.revistacomunicar.com/pdf/2011-04-Delgado.pdfs [Consulta: 1 octubre 2017].

" Fresco-Santalla, Ana María. 2013. Edición y comunicación cientíica: evolución y tendencias actuales. Getafe: Universidad Carlos Ill de Madrid, 2013.109 p. Tesis de maestría. $<$ http://eprints.rclis.org/24471/1/Scholarly_comm_and_publishing_Evolution_and_ Trends_AFresco.pdf 5 [Consulta: 18 marzo 2017].

»Fundación Española para la Ciencia y Tecnología. 2012. Manual de buenas prácticas en edición de revistas científicas. España: Fecyt. p. 33-43. <https://www.revistacomunicar. com/pdf/2013-guia-buenas-practicas.pdf>[Consulta: 6 septiembre 2017].

» García-Aretio, Lorenzo. 2014. OJS y DOI, apuestas por la calidad de las revistas científicas. En RIED. Revista Iberoamericana de Educación a Distancia. Vol. 17, no. 2, 9-13. $<$ http://www.redalyc.org/pdf/3314/331431248001.pdfs[Consulta: 6 septiembre 2017].

» García-Álvarez de Toledo, Juan y René Fernández-Sánchez. 2011. Difusión y divulgación científica en Internet. Asturias: Gobierno del Principado de Asturias, Cienciatec, PCTI Asturias. <https://ria.asturias.es/RIA/bitstream/123456789/1661/1/Archivo. pdf [Consulta: 23 febrero 2017]. 
» González-Vegas, Jesús Alberto. 2010. Visibilidad de las revistas científicas: la experiencia de la revista Vitae una alternativa digital. En Compendium. No. 25, 47-53. <http://www.redalyc.org/html/88o/88019355005> [Consulta: 5 julio 2017].

» Jiménez-Hidalgo, Sonia; Elea Giménez-Toledo y Javier Salvador-Bruna. 2008. Los sistemas de gestión editorial como medio de mejora de la calidad y la visibilidad de las revistas científicas. En El Profesional de la Información. Vol. 17, no. 3, 281-291. <https://doi.org/10.3145/epi.2008.may.04>

» Maricato, João de Melo y Daiane de Brito Fernandes. 2015. Monitoramento de revistas científicas na web com a ferramenta Google Analytics: reflexões a partir da Revista Comunicação \& Informação. En Encontros Bibli: revista eletrônica de biblioteconomia e ciência da informação. Vol. 20, no. 42, 63-78. <http://dx.doi. org/10.5007/1518-2924.2015V2on42p63>

"Mendoza, Sara y Tatiana Paravic. 2006. Origen, clasificación y desafíos de las revistas científicas. En Investigación y Posgrado. Vol. 21, no. 1, 49-75. <http:// www.redalyc.org/articulo.0a?id=65821103>[Consulta: 6 septiembre 2017].

" Miguel, Sandra. 2011. Revistas y producción científica de América Latina y el Caribe: su visibilidad en SciELO, RedALyC y SCOPUS. En Revista Interamericana de Bibliotecología. Vol. 34, no. 2, 187-199. <https://aprendeenlinea. udea.edu.co/revistas/index.php/RIB/article/view/10366/9560>[Consulta: 1 julio 2017].

»Public Knowledge Project. 2016. OJS Reaches 10,000. <https://pkp.sfu. ca/2016/12/15/ojs-reaches-1000o> [Consulta: 6 septiembre 2017].

»Ramírez-Martínez, Diana Cristina; Luis Carlos Martínez-Ruiz y Oscar Fernando Castellanos-Domínguez. 2012. Divulgación, difusión del conocimiento: las revistas científicas. Bogotá: Universidad Nacional de Colombia.<http://www.bdigital.unal.edu.co/8394/1/9789587613346.pdf>[Consulta: 6 septiembre 2017].

»REBIUN. 2011. Ciencia 2.o: aplicación de la web social a la investigación. Madrid: REBIUN. <https://biblioteca.ulpgc.es/files/ciencia_2_o_rebiun_2011. pdf> [Consulta: 25 marzo 2017].

»Robinson-García, Nicolas; Emilio Delgado-López-Cózar y Daniel Torres-Salinas. 2011. Cómo comunicar y diseminar información científica en Internet para obtener mayor visibilidad e impacto. En Aula abierta. Vol. 39, no. 3, 41-50. <http://eprints.rclis.org/28043/1/Dialnet-ComoComunicarYDiseminarInformacionCientificaEnInte-3691479.pdf $>$ [Consulta: 1 abril 2017].

"Rodríguez-Yunta, Luis y Carlos Miguel Tejada-Artigas. 2013. El editor técnico: un perfil necesario para la profesionalización de la edición de revistas científicas en el entorno digital. En Anales de Documentación. Vol. 16, no. 2, 1-9.<http:// dx.doi.org/10.6018/analesdoc.16.2.176391>

"Rojas-V., M. Alejandra y Sandra Rivera-M. 2011. Guía de buenas prácticas para revistas académicas de acceso abierto. Santiago de Chile: ONG Derechos Digitales. <http://www.latindex.org/lat/documentos/descargas/Manual-Buenas_ Practica_Revistas_Academicas.pdf> [Consulta: 5 julio 2017].

»Ruiz-Corbella, Martha y Arturo Galán-González. 2017. La visibilidad de las revistas científicas de educación en el entorno 2.0: el uso de las redes sociales. En Edetania: estudios y propuestas socio-educativas. No. 50, 59-72. <https://dialnet.unirioja.es/servlet/articulo?codigo $=6040100>$ [Consulta: 1 octubre 2017].

» Tejada-Gómez, María Alejandra. 2012. Entre tendencias o disidencias: el futuro de las revistas científicas colombianas. En Unilibros de Colombia. Vol. 19, 108-111. 
$<$ https://www.researchgate.net/publication/304170476_Entre_tendencias_o_ disidencias_El_futuro_de_las_revistas_cientificas_colombianas_Unilibros_ de_Colombia_19_2012_108-111>[Consulta: 1 junio 2018].

»Torres-Salinas, Daniel. 2008a. El paradigma 2.o en las grandes revistas científicas. En 3rd International LIS-EPI Meeting 2008. <http://ec3.ugr.es/publicaciones/ Torres-Salinas,_Daniel-El_paradigma_2_o_en_las_grandes_revistas_cientificas.pdf> [Consulta: 31 marzo 2017].

»Torres-Salinas, Daniel. 2008b. ¿Qué es y cómo se edita una revista científica 2.0? VIII Semana de la Ciencia. Las revistas científicas 2.0 ¿Existen? <http://ec3. ugr.es/publicaciones/Torres-Salinas,_Daniel-Que_es_y_Como_se_edita_una_revista_cientifica.pdfs [Consulta: 1 septiembre 2017].

»Torres-Salinas, Daniel. 2010. Consejos básicos para incrementar la difusión y el impacto de las revistas científicas: métodos honestos y deshonestos. Jornadas para las revistas científicas. <https://es.slideshare.net/torressalinas/consejosbsicos-para-incrementar-la-difusin-y-el-impacto-de-las-revistas-cientficasmtodos-honestos-y-deshonestos> [Consulta: 1 octubre 2017].

»Torres-Salinas, Daniel y Emilio Delgado-López-Cózar. 2009. Estrategia para mejorar la difusión de los resultados de investigación con la Web 2.o. En El Profesional de la Información. Vol. 18, no. 5, 534-539. <https://doi.org/10.3145/ epi.2009.sep.07>

"Uribe-Tirado, Alejandro; Juan Camilo Vallejo-Echavarría y Darío Alexander Betancur- Marín. 2016. Somos visibles y tenemos impacto. Análisis desde datos de acceso abierto, altmetrics y otros de la Revista Interamericana de Bibliotecología. En Revista Interamericana de Bibliotecología. Vol. 39, no. 3, 243275. $\quad<$ https://aprendeenlinea.udea.edu.co/revistas/index.php/RIB/article/ view/323672> [Consulta: 8 octubre 2017].

"Veiga de Cabo, Jorge y Helena Martín-Rodero. 2011. Acceso Abierto: nuevos modelos de edición científica en entornos web 2.o. En Salud Colectiva. Vol. 7, supl. 1. <http://www.scielo.org.ar/scielo.php?script=sci_arttext\&pid=S1851$82652011000300003 \& \operatorname{lng}=e s \& t \mid n g=e s>$ [Consulta: 17 julio 2017]. 